\title{
A novel biliary cannulation method for difficult cannulation cases using a unique, uneven, double-lumen cannula (Uneven method)
}

The utility of pancreatic duct guidewire (P-GW) placement techniques, including the contrast-medium method and the contrast-free wire-guided cannulation method (i.e. the "double-guidewire method" [D-GW]), has been reported for selective biliary cannulation in patients in whom performing cannulation of the bile duct is difficult [1-5]. However, P-GW placement often disturbs the approach to the bile orifice. Moreover, it is sometimes difficult to insert the catheter or guidewire in the direction of the bile duct, which delays biliary cannulation.

Herein, we report a novel biliary cannulation method using a unique, uneven, double-lumen cannula (UDLC; PIOLAX, Tokyo, Japan). The UDLC is a double-lumen catheter, with lumens measuring 0.025 and 0.035 inches in diameter, respectively. The orifice of each lumen is uneven, thereby forming a channel at the tip of the UDLC ( $\triangleright$ Fig.1). With such characteristics in mind, we applied the UDLC to develop a new method of selec- tive biliary cannulation for difficult cannulation cases (UDLC method). We describe a case wherein the UDLC was successfully employed without complications ( $\triangleright$ Video 1 ).

A 75-year-old man presented with pancreatic cancer and was admitted to our hospital for treatment of obstructive cholangitis. It was difficult to perform biliary cannulation as we could insert only the P-GW. Initially, the UDLC was used to intubate the papilla through the P-GW via the distal lumen. This straightened the pancreatic duct and the common channel, thereby effectively stabilizing the papilla ( $\triangleright$ Fig. 2). Next, we performed biliary cannulation via the proximal lumen, as is done in the D-GW method (> Fig.3). By using this method, we were able to avoid the time delay in adjusting the catheter axis to comply with the bile duct direction, as required in the P-GW method. Thus, we easily initiated the biliary cannulation approach. Ultimately, we succeeded in performing selective biliary cannulation ( $>$ Fig. 4 ).

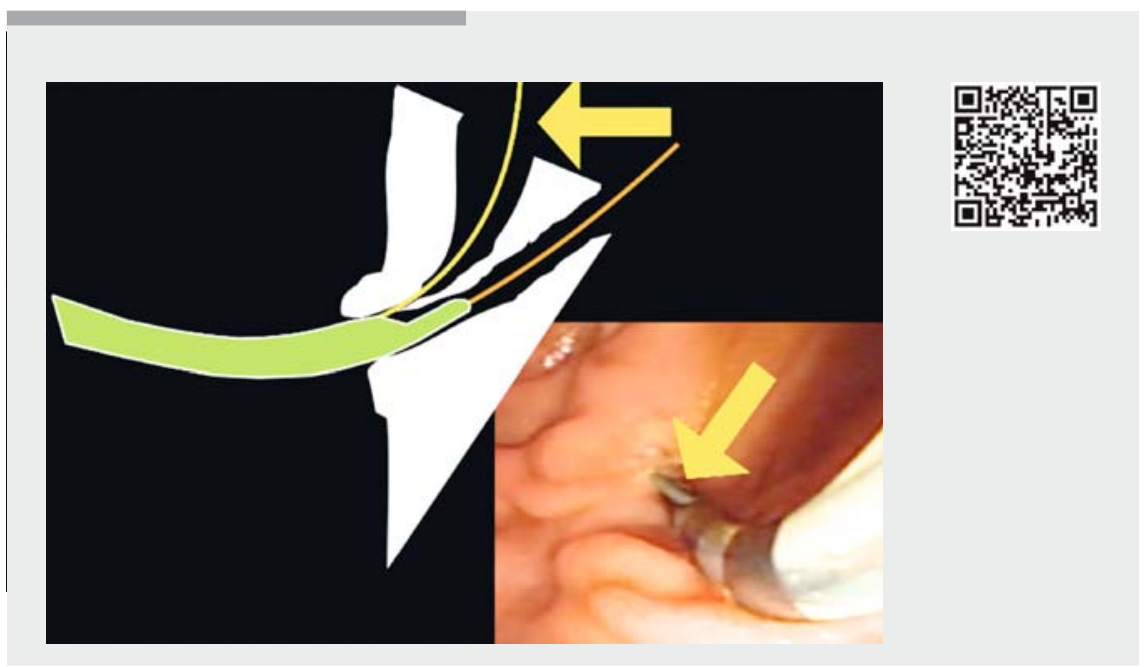

$\checkmark$ Video 1 The uneven double-lumen cannula (UDLC) method is used for cases of difficult cannulation. The papilla is intubated using the UDLC, then biliary cannulation via the proximal lumen is quickly performed.

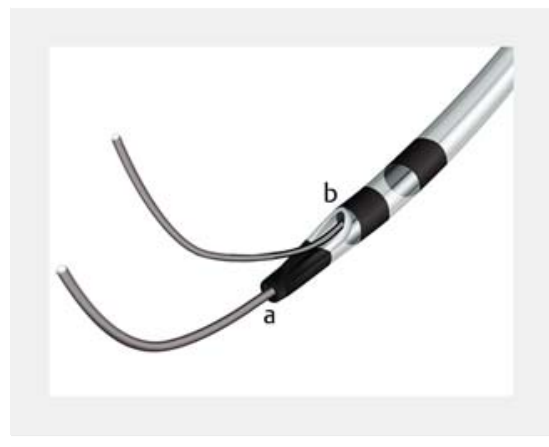

Fig. 1 The uneven double-lumen cannula (UDLC; PIOLAX, Tokyo, Japan) is a double-lumen catheter, with lumens of 0.025 (distal, a) and 0.035 (proximal, b) inches in diameter. The orifice of each lumen is uneven, thereby creating a channel within the tip.

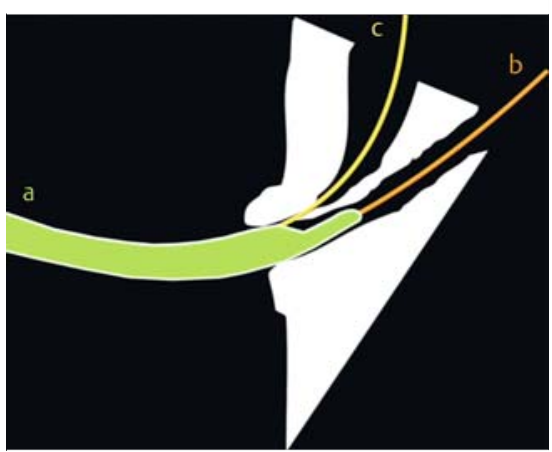

- Fig. 2 A schematic of the uneven double-lumen cannula (UDLC) method. Initially, the UDLC $\mathbf{a}$ is intubated to the papilla through the pancreatic guidewire (P-GW) b using the distal lumen. This straightens the pancreatic duct and the common channel, thereby more effectively stabilizing the papilla compared with the use of the P-GW alone. Next, biliary cannulation using the proximal lumen is performed in a manner that is similar to the double-guidewire technique $\mathbf{c}$. 


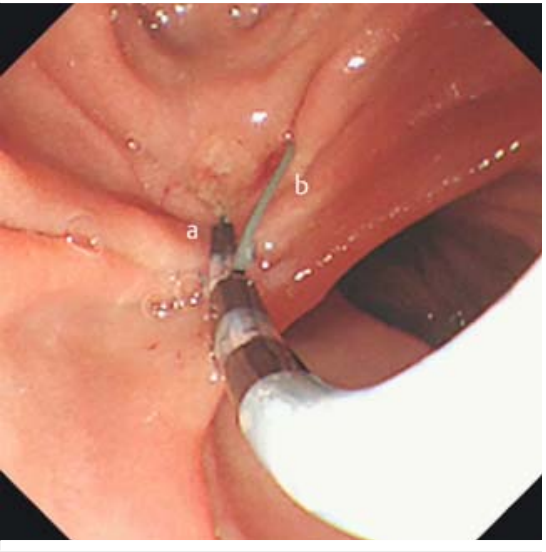

- Fig. 3 The tip of the uneven doublelumen cannula a is intubated to the papilla through the pancreatic guidewire. The guidewire from the proximal lumen b is seen.

In summary, we report a new cannulation method using a UDLC to safely and effectively perform selective biliary cannulation in patients in whom biliary cannulation is otherwise difficult.

Endoscopy_UCTN_Code_CCL_1AZ_2AI

\section{Competing interests}

None

The authors

Mamoru Takenaka ${ }^{1,2}$, Yoshifumi Arisaka ${ }^{1,3}$, Arata Sakai ${ }^{1}$, Takashi Kobayashi ${ }^{1}$, Hideyuki Shiomi ${ }^{1}$, Atshuhiro Masuda ${ }^{1}$, Masatoshi

\section{Kudo $^{2}$}

1 Department of Gastroenterology, Kobe University Faculty of Medicine, Hyogo, Japan

2 Department of Gastroenterology and Hepatology, Kindai University Faculty of Medicine, Osaka-Sayama, Japan

3 Department of Gastroenterology, Nissay Hospital, Osaka, Japan
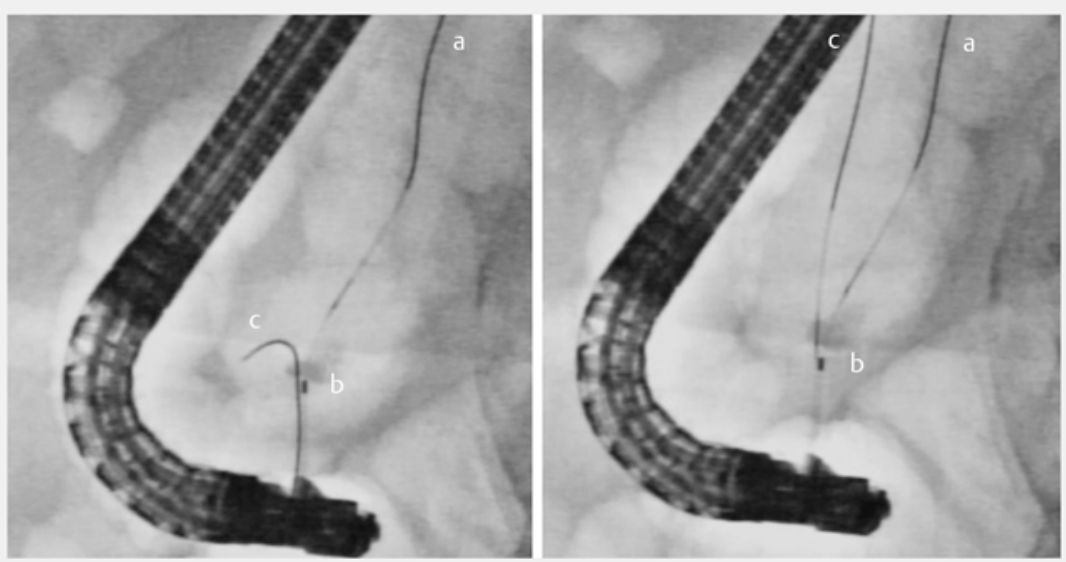

- Fig. 4 The uneven double-lumen cannula (UDLC) is intubated to the papilla through the pancreatic guidewire $\mathbf{a}$. The fluoroscopic marker of the UDLC $\mathbf{b}$ is seen. The guidewire from the proximal lumen $\mathbf{c}$ is used for biliary cannulation, as is done in the double-guidewire method.

\section{Corresponding author}

\section{Mamoru Takenaka, MD, PhD}

Department of Gastroenterology and Hepatology, Kindai University Faculty of Medicine, 377-2 Ohno-Higashi, OsakaSayama, 589-8511, Japan Fax: +81-72-3672880

mamoxyo45@gmail.com

\section{References}

[1] Dumonceau JM, Devière J, Cremer M. A new method of achieving deep cannulation of the common bile duct during endoscopic retrograde cholangiopancreatography. Endoscopy 1998; 30: S80

[2] Maeda S, Hayashi H, Hosokawa O et al. Prospective randomized pilot trial of selective biliary cannulation using pancreatic guidewire placement. Endoscopy 2003; 35: 721 724

[3] Ito K, Fujita N, Noda Y et al. Pancreatic guidewire placement for achieving selective biliary cannulation during endoscopic retrograde cholangio-pancreatography. World J Gastroenterol 2008; 14: 5595-5600

[4] Herreros de Tejada A, Calleja JL, Diaz G et al. Double-guidewire technique for difficult bile duct cannulation: a multicenter randomized, controlled trial. Gastrointest Endosc 2009; 70: 700 - 709

[5] Gronroos JM, Vihervaara H, Gullichsen R et al. Double-guidewire-assisted biliary cannulation: experiences from a single tertiary referral center. Surg Endosc 2011; 25: 15991602

\section{Bibliography}

DOI https://doi.org/10.1055/a-0624-9317

Published online: 12.6.2018

Endoscopy 2018; 50: E229-E230

(C) Georg Thieme Verlag KG

Stuttgart · New York

ISSN 0013-726X

\section{ENDOSCOPY E-VIDEOS}

https://eref.thieme.de/e-videos

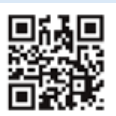

Endoscopy E-Videos is a free access online section, reporting on interesting cases and new

techniques in gastroenterological endoscopy. All papers include a high quality video and all contributions are freely accessible online.

This section has its own submission website at

https://mc.manuscriptcentral.com/e-videos 